\title{
Intrinsic point defects and the $n$ - and $p$-type dopability of the narrow gap semiconductors GaSb and InSb
}

\author{
J. Buckeridge,,${ }^{1, *}$ T. D. Veal, ${ }^{2}$ C. R. A. Catlow, ${ }^{1}$ and D. O. Scanlon ${ }^{1,3,4}$ \\ ${ }^{1}$ Department of Chemistry, University College London, \\ 20 Gordon Street, London WC1H OAJ, United Kingdom \\ ${ }^{2}$ Stephenson Institute for Renewable Energy and Department of Physics, School of Physical Sciences, \\ University of Liverpool, Liverpool L69 7ZF, United Kingdom \\ ${ }^{3}$ Diamond Light Source Ltd., Diamond House, Harwell Science and Innovation Campus, Didcot, Oxfordshire OX11 ODE, United Kingdom \\ ${ }^{4}$ Thomas Young Centre, University College London, Gower Street, London WC1E 6BT, United Kingdom
}

(Received 24 May 2019; published 25 July 2019)

\begin{abstract}
The presence of defects in the narrow gap semiconductors $\mathrm{GaSb}$ and $\mathrm{InSb}$ affects their dopability and hence applicability for a range of optoelectronic applications. Here, we report hybrid density functional theory (DFT)-based calculations of the properties of intrinsic point defects in the two systems, including spin-orbit coupling effects, which influence strongly their band structures. With the hybrid DFT approach adopted, we obtain excellent agreement between our calculated band dispersions and structural, elastic, and vibrational properties and available measurements. We compute point defect formation energies in both systems, finding that antisite disorder tends to dominate, apart from in GaSb under certain conditions, where cation vacancies can form in significant concentrations. Calculated self-consistent Fermi energies and equilibrium carrier and defect concentrations confirm the intrinsic $n$ - and $p$-type behavior of both materials under anion-rich and anion-poor conditions. Moreover, by computing the compensating defect concentrations due to the presence of ionized donors and acceptors, we explain the observed dopability of GaSb and InSb.
\end{abstract}

DOI: 10.1103/PhysRevB.100.035207

\section{INTRODUCTION}

$\mathrm{GaSb}$ and InSb belong to the family of III-V, zinc-blende structured semiconductors of interest from both fundamental and technological points of view. The incorporation of $\mathrm{Sb}$ in III-V semiconducting nitrides, phosphides, and arsenides results in a redshift of the band gap, opening up the possibility of pushing the frequency domain of devices based on such materials far into the infrared (IR) [1-3]. Both GaSb and InSb have applications in long-wavelength telecommunications [4], high-speed microelectronics [5-7], and optoelectronics [8,9]. Due to favorable lattice matching, GaSb can be used as a substrate for a wide range of ternary and quaternary III-V compounds [10-13]. The spin-orbit interaction (SOI) has a strong effect on the valence band structure of both systems [14-16] but is more pronounced in InSb [17,18], which, combined with a large Landé $g$ factor (over 50) [19], has meant that InSb has attracted considerable attention in the field of Majorana physics [20,21]. Moreover, GaSb and InSb have both been demonstrated to incorporate $\mathrm{N}$ and $\mathrm{Bi}$ effectively, resulting in a reduction in band gap [22-38] in a manner similar to the more widely studied, GaAs-based dilute nitrides and bismides [39,40]. Alloys can be produced of GaAs, GaSb, and InSb, together with the relevant nitrides and/or bismides to tune the optical and electronic properties for a variety of applications [41-45]; indeed, very high efficiency tandem

\footnotetext{
*j.buckeridge@ucl.ac.uk
}

solar cells include an active layer composed of such an alloy [46].

Given the importance of $\mathrm{GaSb}$ and $\mathrm{InSb}$, there are surprisingly few studies on their intrinsic defect properties, which are key to their dopability and hence functionality in devices. As-grown $\mathrm{GaSb}$ has been shown to be $p$-type regardless of growth conditions [12,16,47-50], although the acceptor concentrations can be decreased slightly by varying the $\mathrm{V} / \mathrm{III}$ flux when growing with molecular beam epitaxy (MBE) [51,52]. Gallium vacancies, $V_{\mathrm{Ga}}$, have been shown to occur in $\mathrm{GaSb}$ using positron annihilation spectroscopy (PAS) [53] but have been ruled out as the dominant acceptor; instead, it has been inferred in further PAS studies that the gallium antisite $(\mathrm{Ga} s \mathrm{sb})$ is responsible for the observed $p$-type activity $[54,55]$ based on earlier density functional theory (DFT) calculations using the local-density approximation (LDA) [56]. While the LDA was also used to investigate the role of $\mathrm{H}$ in GaSb [57], this approach suffers from the well-known band gap underestimation error, which is particularly problematic in narrow gap semiconductors such as $\mathrm{GaSb}$ and InSb. To overcome the band gap error, a subsequent study on defects in GaSb employed hybrid DFT (without including the SOI) [58]. The results, however, indicated that the intrinsic defect physics would result in a semi-insulating material as grown, in contrast to experiment. $\mathrm{C}$ and $\mathrm{O}$ impurities were instead proposed to account for the $p$-type activity.

There are even fewer studies of the defect properties of InSb. The material can be made $n$ or $p$ type depending on growth conditions, while temperature-( $T)$ dependent studies 
have been employed to study variations in the $n$-type carrier concentration, Fermi energy, and mobilities in order to elucidate various defect properties [50,59-63]. A computational study using DFT with the LDA indicated that the antimony antisite $\left(\mathrm{Sb}_{\text {In }}\right)$ would dominate in $\mathrm{Sb}$-rich growth conditions [64]; by varying growth conditions, it was suggested that the formation of this defect could be suppressed in epitaxially grown thin films [63]. Furthermore, it has been proposed that the formation of indium vacancies as well as $\mathrm{Sb}_{\text {In }}$ can account for observed changes in the electronic properties of InSb grown in varying conditions [65]. To our knowledge, no comprehensive study on the intrinsic defects in InSb using hybrid DFT has yet been performed.

In this paper, we use hybrid DFT, including the SOI, to investigate the dominant native point defects in both $\mathrm{GaSb}$ and InSb. As noted above, the SOI strongly affects the dispersion of the upper valence bands in both systems; therefore, depending on the composition of the particular defect states, it can have a significant effect on the defect formation energies. We tune the fraction of exact exchange in the hybrid functional to reproduce only the band gaps and justify this approach by computing a range of bulk properties of both systems, demonstrating close agreement with experiment for the structural, electronic, elastic, and lattice vibrational properties. Our results show that $\mathrm{GaSb}$ will be $p$ type when grown in Sb-poor conditions but may be semi-insulating under Sb-rich conditions. InSb, in contrast, will be $n$ type under $\mathrm{Sb}$-poor conditions and $p$ type under $\mathrm{Sb}$-rich conditions. From our computed defect formation energies, we determine self-consistent Fermi energies and equilibrium carrier and defect concentrations as a function of $T$ by imposing the constraint of charge neutrality, calculating concentrations that agree well with experiment. Moreover, by introducing fixed concentrations of fully ionized dopants into the self-consistent Fermi energy calculation, we investigate donor and acceptor compensation by native defects in both systems. We find that, while InSb can be easily $n$ or $p$ doped, GaSb cannot be effectively $n$ doped under Sb-poor conditions. We provide the first comprehensive study of intrinsic disorder in $\mathrm{GaSb}$ and InSb using relativistic hybrid DFT, which helps to elucidate the defect properties and dopability of both systems under equilibrium conditions.

The rest of the paper is structured as follows: In Sec. II, we describe our computational methodology. We present our results in Sec. III and summarize our main findings in Sec. IV.

\section{CALCUlations}

To calculate the bulk and defect properties of GaSb and InSb, we have used plane-wave DFT as implemented in the VASP code [66-69], utilizing the Heyd-Scuseria-Ernzerhof (HSE06) hybrid density functional [70] for electron exchange and correlation with the projector augmented-wave method [71] to model the interaction between core and valence electrons (including $3 d$ and $4 d$ states among the 13 valence electrons in the cases of Ga and In, respectively, and 5 valence electrons for As). Spin-orbit interactions were included in all calculations [72]. The proportion $\alpha$ of exact exchange in the hybrid functional was set to $\alpha=0.335$ ( $\alpha=0.31$ ) for $\mathrm{GaSb}(\mathrm{InSb})$ in order to reproduce the fundamental gap (see below). The total energy of the zinc-blende primitive cell was calculated at a series of constant volumes, using a $400 \mathrm{eV}$ plane-wave cutoff and a $12 \times 12 \times 12 \Gamma$-centered MonkhorstPack [73] $k$-point mesh [a finer $14 \times 14 \times 14 k$-point grid was used when computing the density of states (DOS)], which provided convergence in the total energy up to $10^{-4} \mathrm{eV}$, fitting the resultant energy-volume data to the Murnaghan equation of state. The bulk modulus $B_{0}$ was derived using this approach. The zone-center longitudinal phonon frequencies $\omega_{\mathrm{LO}}$ were calculated using the frozen-phonon approach, as implemented in VASP [74]. We have also computed the elastic constants $C_{11}, C_{12}$, and $C_{44}$, using the finite-displacement approach available in VASP. Electron $\left(m_{\mathrm{e}}^{*}\right)$, light-hole $\left(m_{\mathrm{lh}}^{*}\right)$, and heavy-hole $\left(m_{\mathrm{hh}}^{*}\right)$ effective masses were calculated by fitting quadratic functions to the energy dispersion within $1 \mathrm{meV}$ of the appropriate band extremum. For the hole masses, derived from the valence bands where the dispersion is nonspherical, we took an average of the values obtained for the different Cartesian directions.

Defect calculations were performed using the supercell approach with a 64-atom $2 \times 2 \times 2$ expansion of the conventional cubic cell, which was shown to be suitably converged previously $[36,57,58,75-77]$. The formation energy of defect $X$ in charge state $q, E_{f}\left(X^{q}\right)$, was determined through calculation of the heat of formation of the relevant defect reaction [78,79]:

$$
\begin{aligned}
E_{f}\left(X^{q}\right)= & E_{\mathrm{tot}}\left(X^{q}\right)-E_{\mathrm{tot}}(\mathrm{bulk})-\sum_{i} n_{i} \mu_{i} \\
& +q\left(E_{\mathrm{VBM}}+\Delta+E_{F}\right)+E_{\mathrm{c}}
\end{aligned}
$$

where $E_{\text {tot }}\left(X^{q}\right)\left[E_{\text {tot }}(\right.$ bulk $\left.)\right]$ is the total energy of the defectcontaining (pure bulk) supercell, $E_{\mathrm{VBM}}$ is the energy at the valence band maximum (VBM), $E_{F}$ is the Fermi energy (introduced as a parameter), $\Delta$ is the energy required to align the electrostatic potential in the defect supercell with that of bulk, and $E_{\mathrm{c}}$ is a correction term to account for supercell errors such as image charge interactions and, where applicable, erroneous band filling by delocalized carriers. To calculate $\Delta$ and $E_{\mathrm{c}}$, we follow the procedure outlined by Lany and Zunger [80], which has been shown to result in corrections closely matched to those derived from full solutions to Poisson's equation [81]. $n_{i}$ is the number of species $i$ that is added to $\left(n_{i}>0\right)$ or removed from $\left(n_{i}<0\right)$ the supercell to form $X$, and $\mu_{i}$ is the chemical potential of species $i$, taken with reference to the calculated standard state energies $E_{i}$ so that $\mu_{i}=E_{i}+\Delta \mu_{i}$ [82]. The values of $\Delta \mu_{i}$ can vary depending on the environmental conditions in thermodynamic equilibrium but are constrained by the relation $\Delta \mu_{\mathrm{M}}+\Delta \mu_{\mathrm{Sb}}=\Delta H[M \mathrm{Sb}]$, where $M=\mathrm{Ga}$ or In and $\Delta H[M \mathrm{Sb}]$ is the heat of formation of $M \mathrm{Sb}$; we calculate $\Delta H[\mathrm{GaSb}]=-0.507 \mathrm{eV}$ and $\Delta H[\mathrm{InSb}]=-0.470 \mathrm{eV}$, which are in reasonable agreement with the experimental values of -0.433 and $-0.316 \mathrm{eV}$, respectively [83], particularly taking into account that the experimental values correspond to room $T$, while the calculations are done at the athermal limit (one would expect the heats of formation to become more negative by $\sim 0.05 \mathrm{eV}[83]$ at $0 \mathrm{~K}$ ) [84]. We calculate $E_{f}[X]$ at two extremes: $\mathrm{Sb}$ rich, where $\Delta \mu_{\mathrm{Sb}}=0 \mathrm{eV}$, corresponding to an excess of $\mathrm{Sb}$ in the growth environment and the absence 
TABLE I. Calculated lattice parameter $a$; bulk modulus $B_{0}$; elastic constants $C_{11}, C_{12}$, and $C_{44}$; band gap $E_{g}$; spin-orbit split-off energy $\Delta_{\mathrm{SO}}$; electron $\left(m_{\mathrm{e}}^{*}\right)$, light-hole $\left(m_{\mathrm{lh}}^{*}\right)$, and heavy-hole $\left(m_{\mathrm{hh}}^{*}\right)$ effective masses; and zone-center longitudinal optical phonon frequency $\omega_{\mathrm{LO}}$ of $\mathrm{GaSb}$ and InSb, compared with experimental results [59,89-101]. The effective masses are given in units of the electronic rest mass.

\begin{tabular}{|c|c|c|c|c|c|c|c|c|c|c|c|}
\hline & $a(\AA)$ & $B_{0}(\mathrm{GPa})$ & $\mathrm{C}_{11}(\mathrm{GPa})$ & $\mathrm{C}_{12}(\mathrm{GPa})$ & $\mathrm{C}_{44}(\mathrm{GPa})$ & $E_{g}(\mathrm{eV})$ & $\Delta_{\mathrm{SO}}(\mathrm{eV})$ & $m_{\mathrm{e}}^{*}$ & $m_{\mathrm{lh}}^{*}$ & $m_{\mathrm{hh}}^{*}$ & $\omega_{\mathrm{LO}}\left(\mathrm{cm}^{-1}\right)$ \\
\hline \multicolumn{12}{|l|}{$\mathrm{GaSb}$} \\
\hline Calc. & 6.137 & 55.1 & 92.33 & 39.03 & 45.99 & 0.808 & 0.76 & 0.041 & 0.047 & 0.23 & 230.4 \\
\hline Expt. & 6.09593 [89] & 56.35 [90] & 90.82 [91] & 41.31 [91] & 44.47 [91] & 0.813 [92] & 0.82 [93] & 0.0412 [94] & 0.05 [95] & 0.28 [95] & $232.6[96]$ \\
\hline \multicolumn{12}{|l|}{$\mathrm{InSb}$} \\
\hline Calc. & 6.548 & 40 & 68.2 & 33.8 & 31.6 & 0.23 & 0.80 & 0.018 & 0.019 & 0.25 & 180.3 \\
\hline Expt. & 6.4794 [97] & 48.1 [98] & 69.18 [98] & 37.88 [98] & 31.32 [98] & 0.24 [99] & 0.80 [99] & 0.015 [59] & 0.015 [100] & $0.43[100]$ & 196.8 [101] \\
\hline
\end{tabular}

of pure In, and $\mathrm{Sb}$ poor, the opposite extreme, where $\Delta \mu_{\mathrm{Sb}}=$ $\Delta H[M \mathrm{Sb}]$.

From the calculated defect formation energies and DOS, we used the code SC-FERMI [85-88] to determine the equilibrium carrier and defect concentrations. SC-FERMI employs Fermi-Dirac statistics to calculate the concentrations, which are functions of $E_{F}$. With the constraint of overall charge neutrality in the system, a self-consistent $E_{F}$ can be derived at any temperature, and consequently, so can the electron $\left(n_{0}\right)$, hole $\left(p_{0}\right)$, and defect $([X])$ concentrations. Moreover, the charge neutrality constraint can be exploited in order to introduce fixed concentrations of ionized impurities and the equilibrium carrier and defect concentrations recalculated in the presence of such impurities. In such a way, one can analyze ionized donor and acceptor compensation. In our calculations, we neglect the temperature dependence of the free energies of defect formation due to the high computational cost in determining the associated vibrational entropy; one would expect the free energies to change by $\sim 0.1-0.2 \mathrm{eV}$ over the temperature range we employ, but including such changes would not significantly affect the conclusions we draw from our results.

\section{RESULTS}

\section{A. Bulk properties}

In Table I, we show our calculated lattice parameter $a ; B_{0}$; elastic constants $C_{11}, C_{12}$, and $C_{44}$; band gap $E_{g}$; spin-orbit split-off energy $\Delta_{\mathrm{SO}} ; m_{\mathrm{e}}^{*}, m_{\mathrm{lh}}^{*}$, and $m_{\mathrm{hh}}^{*}$; and $\omega_{\mathrm{LO}}$ for $\mathrm{GaSb}$ and InSb, compared with experiment [59,89-101]. As described above, the $\alpha$ used in the hybrid functional was chosen to reproduce the band gap at low $T$. From Table I, however, we see that the hybrid DFT approach reproduces very well the experimental structural, elastic, and lattice vibrational properties of both materials, while the energy-dispersion-derived properties are also well reproduced. The only significant discrepancies occur for InSb, particularly in $B_{0}$ and $\omega_{\mathrm{LO}}$, indicating a slightly softer lattice in the calculation compared with experiment. The calculated $m_{\mathrm{hh}}^{*}$ for InSb is significantly lower than the experimental value, but this discrepancy may be due to difficulties in measuring this property accurately. Overall, the agreement between the calculated values and experiment is satisfactory and indicates that our DFT approach is appropriate.

In Fig. 1, we show our hybrid-DFT-computed band structures of $\mathrm{GaSb}$ and $\mathrm{InSb}$ compared with experimental values determined using angle-resolved photoemission spectroscopy and, for the case of $\mathrm{GaSb}$, reflectance measurements [93, 102-104]. For GaSb, we have also calculated band energies using the fully self-consistent $G W$ approach, as implemented in VASP [105-107], including the SOI. As these calculations

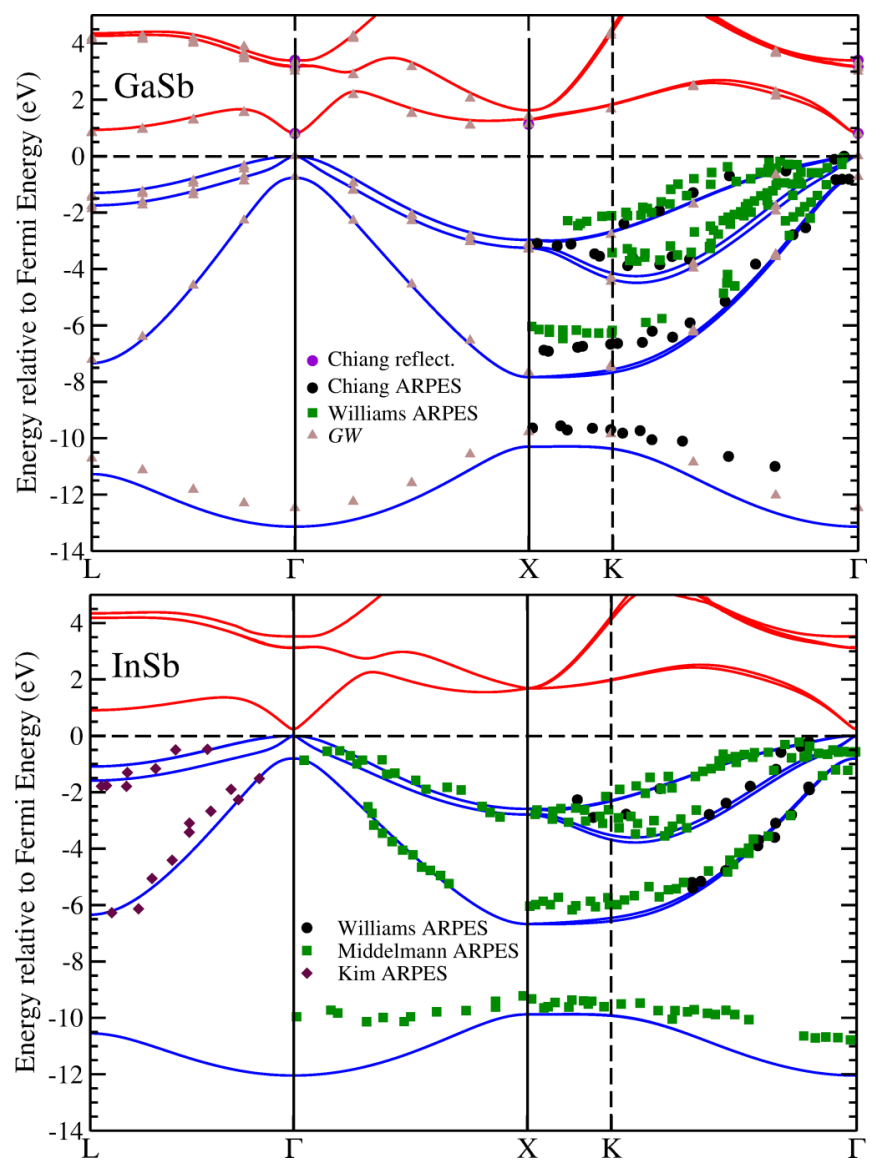

FIG. 1. Band structure of GaSb and InSb calculated using hybrid density functional theory (valence bands are indicated by blue lines; conduction bands are indicated by red lines), compared with experimental results determined for the case of $\mathrm{GaSb}$ using reflectance measurements by Chiang and Eastman [93] (purple circles) and angle-resolved photoemission spectroscopy (ARPES; black circles and green squares) by Chiang and Eastman [93] and Williams et al. [102], as well as calculated energy levels using self-consistent $G W$ (brown triangles). The InSb bands are compared with ARPES measurements by Williams et al. [102] (black circles), Middelmann et al. [103] (green squares), and Kim et al. [104] (maroon diamonds). 


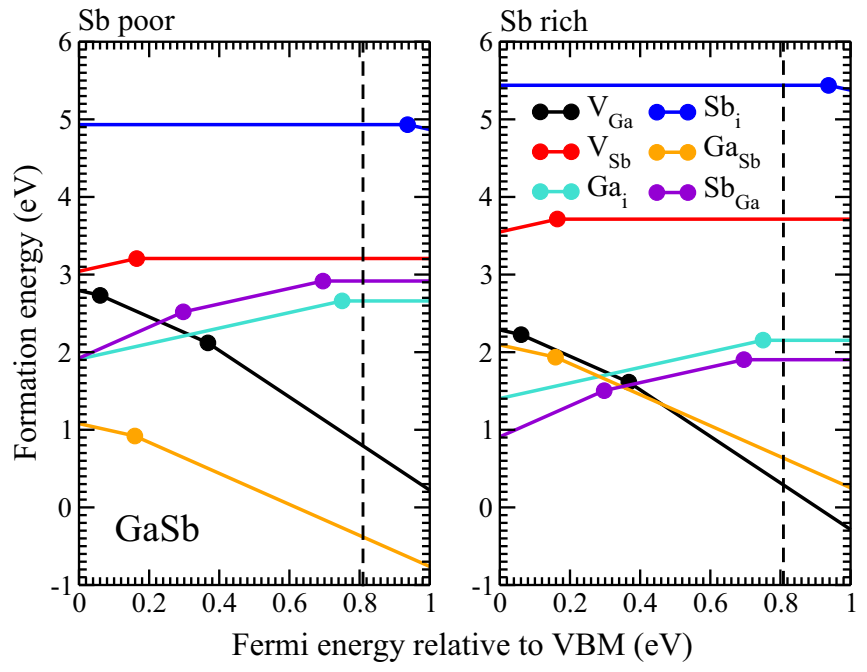

FIG. 2. Calculated formation energies of each intrinsic defect (vacancies, interstitials, and antisites; see text for description) in $\mathrm{GaSb}$ as a function of Fermi energy relative to the valence band maximum (VBM), shown for Sb-poor and Sb-rich conditions. The slope of each line indicates the defect charge state; the transition levels lie where the slopes change. The dashed line indicates the position of the conduction band minimum.

are computationally expensive, we have not determined the dispersion along the high-symmetry path in the Brillouin zone with as small a grid spacing as we have for the hybrid DFT calculations. The band structure is similar in both cases to GaAs [108], with the VBM and conduction band minimum (CBM) both occurring at the $\Gamma$ point and a splitting of the sixfold-degenerate upper valence bands into fourfold- and twofold-degenerate bands, the latter forming the spin-orbit split-off bands. For both systems, the hybrid DFT approach reproduces the band structure well, apart from the lower-lying $\mathrm{Sb} s$ states (at about $-11 \mathrm{eV}$ ), which are deeper than either experiment or the $G W$ results. The bands near the VBM and the CBM, however, are very well reproduced. These bands are the most significant for defect state formation.

\section{B. Defects in GaSb}

Our calculated formation energies of intrinsic defects in $\mathrm{GaSb}$ are shown in Fig. 2 as a function of $E_{F}$, referenced to the VBM, for Sb-poor and Sb-rich conditions. Gasb dominates in Sb-poor conditions; it has a formation energy under $1 \mathrm{eV}$ and is negatively charged for all values of $E_{F}$ within the band gap, with an adiabatic transition from the - to 2state $(-/ 2-)$, occurring at $E_{F}=0.16 \mathrm{eV}$ above the VBM. Such a low-energy, negatively charged defect indicates an intrinsically $p$-type material, as is observed experimentally [12,16,47-49]. All other defects have formation energies of at least $1 \mathrm{eV}$ higher than $\mathrm{Ga}_{\mathrm{Sb}}$ for $E_{F}$ within the band gap. Previous calculations by Hakala et al., using DFT-LDA [56], and Virkkala et al. [58], using hybrid DFT, both found that $\mathrm{Ga}_{\mathrm{Sb}}$ had the lowest formation energy for $E_{F}$ in the upper half of the band gap but predicted compensation by Ga interstitials $\left(\mathrm{Ga}_{i}^{+}\right)$, resulting in an insulating material. The LDA calculations did not include the SOI or any correction for the band gap underestimation, while the hybrid DFT calculations did not include the SOI and used higher-convergence criteria than those we employ [58]; their results contradict the experimentally observed $p$-type activity of undoped GaSb.

In Sb-rich conditions, we find that $E_{f}\left(\mathrm{Ga}_{\mathrm{Sb}}\right)$ increases significantly, while $E_{f}\left(V_{\mathrm{Ga}}\right)$ and $E_{f}\left(\mathrm{Sb}_{\mathrm{Ga}}\right)$ both decrease, so that the lowest-energy defects are $\mathrm{Sb}_{\mathrm{Ga}}$ for $E_{F}<0.36 \mathrm{eV}$ and $V_{\mathrm{Ga}}$ for $E_{F}>0.42 \mathrm{eV}$, with $\mathrm{Ga}_{\mathrm{Sb}}$ having the lowest energy for $E_{F}$ between these ranges. $\mathrm{As} \mathrm{Sb}_{\mathrm{Ga}}$ are positively charged and $\mathrm{Ga}_{\mathrm{Sb}}$ and $V_{\mathrm{Ga}}$ are negatively charged for $E_{F}$ within the band gap, these defects self-compensate, and one would expect $E_{F}$ to remain trapped roughly midgap, resulting in an intrinsically insulating material (we note that the formation energy of $\mathrm{Ga}_{i}$ is also low in this range of $E_{F}$, and we expect that this defect will play a minor role in the self-compensation mechanism). These formation energies suggest significant concentrations of $V_{\mathrm{Ga}}$ will be present, in agreement with PAS studies [53-55,109], but the insulating nature contradicts the $p$-type activity of $\mathrm{GaSb}$ observed in many differently produced samples. It may be the case that, in nonequilibrium growth techniques, formation of the compensating $\mathrm{Sb}_{\mathrm{Ga}}$ may be suppressed, which would result in a $p$-type material where the hole concentration arises from the ionization of $V_{\mathrm{Ga}}$ and $\mathrm{Ga}_{\mathrm{Sb}}$ [51,52]. Our results for $\mathrm{Sb}$-rich conditions agree qualitatively with those of Virkkala et al. [58], although they did not predict that the $V_{\mathrm{Ga}}$ would become the lowest-energy defect for any value of $E_{F}$ within the band gap. Comparisons with the LDA calculations of Hakala et al. [56] are more difficult, as they only reported formation energies for $\mathrm{Sb}_{\mathrm{Ga}}$ in the neutral state. We note, however, that they also found $V_{\mathrm{Ga}}$ to be the lowest-energy defect close to the CBM.

From our computed defect formation energies and total DOS, we have calculated the self-consistent $E_{F}$ and equilibrium carrier and defect concentrations by applying the constraint of overall charge neutrality to our system. The results are shown in Fig. 3(a) over the $T$ range below the melting point (985 K [83]). It is worth noting here that, when varying $T$ in this analysis and for the case of InSb below, we do not take into account the variation in band gap, which can be substantial for these narrow gap semiconductors. Indeed, at room temperature the band gap reduces by $86 \mathrm{meV}$ for $\mathrm{GaSb}$ [1] and $67 \mathrm{meV}$ for InSb [99], compared with their extrapolated $0 \mathrm{~K}$ values. Such reductions are a result of thermal expansion and increased electron-phonon coupling, the modeling of which is beyond the scope of this study on defects in both systems. Including the experimental variation in $E_{g}$ with $T$ in our calculations is not straightforward, as the defect transition levels vary with $T$ in a nontrivial manner. If we do include just the experimental $E_{g}$ variation, we calculate slightly different electron and hole concentrations which do not alter our conclusions significantly. As modeling temperature effects on the defect formation and transition levels is beyond the scope of the current work, we present our analysis below with the band gap fixed for all temperatures studied. We expect that, at higher $T$, where the band gap is reduced and, consequently, the electron and hole concentrations are increased, compensating defect formation energies will also be lowered as vibrational entropy contributions to the free energy become more significant, so that the changes in concentrations will approximately cancel each other. 


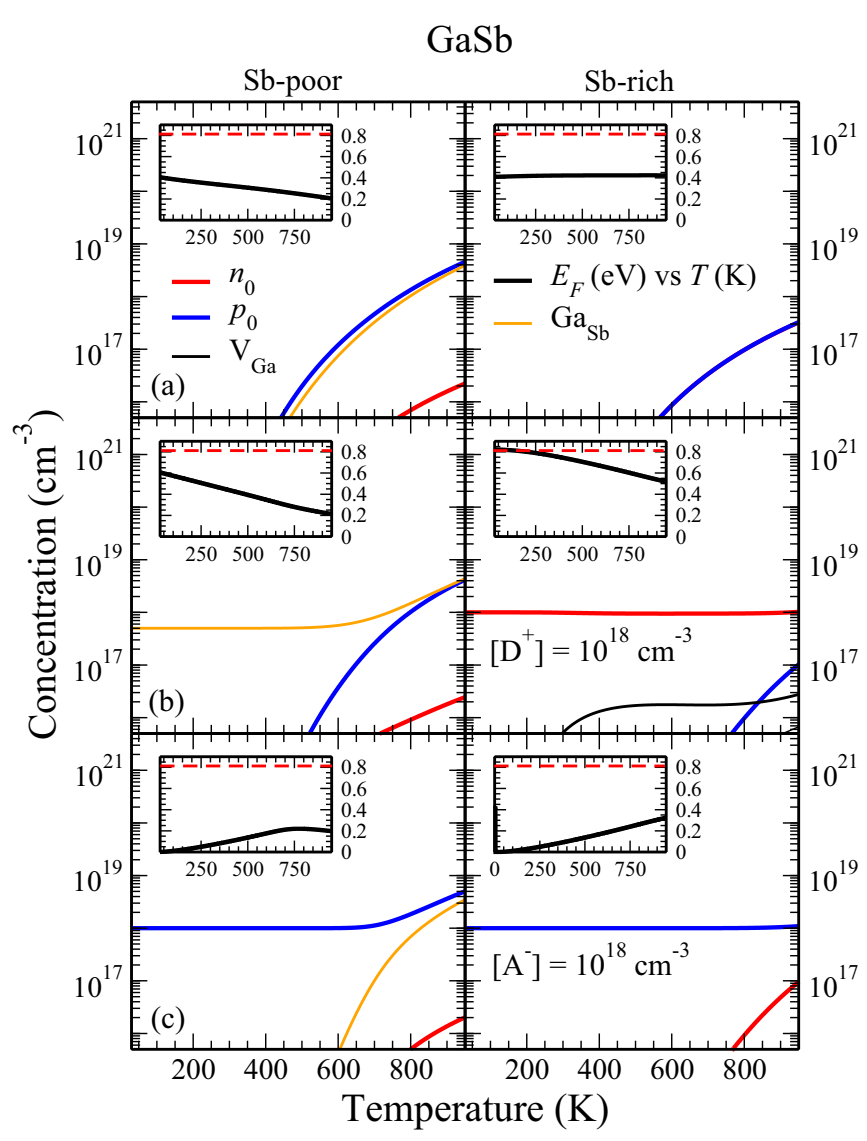

FIG. 3. Concentrations of electron $\left(n_{0}\right)$ and hole $\left(p_{0}\right)$ carriers and defects (vacancies, interstitials, and antisites; see text for description) in $\mathrm{GaSb}$ as a function of temperature $T$ calculated (a) for equilibrium conditions, (b) in the presence of a fixed concentration of donors $\left[\mathrm{D}^{+}\right]=10^{18} \mathrm{~cm}^{-3}$, and (c) for a fixed concentration of acceptors $\left[\mathrm{A}^{-}\right]=10^{18} \mathrm{~cm}^{-3}$. The results are shown for Sb-poor and Sb-rich conditions in the left and right panels, respectively. The insets show the self-consistent Fermi energy $E_{F}$ as a function of $T$, with the conduction band minimum indicated by the red dashed line.

From our analysis we find that, in Sb-poor conditions, $\mathrm{GaSb}$ is $p$ type with hole concentrations $p_{0}$ of $\sim 10^{16}-10^{18}$ $\mathrm{cm}^{-3}$ for $400<T<800 \mathrm{~K}$. The source of $p_{0}$ is the formation and ionization of $\mathrm{Ga}_{\mathrm{Sb}} ; p_{0}$ is equal to $2\left[\mathrm{Ga}_{\mathrm{Sb}}\right]$, which is consistent with the dominant charge state of $\mathrm{Ga}_{\mathrm{Sb}}$ being 2-, but at $T \approx 800 \mathrm{~K}$ the concentrations come close to being equal, as $E_{F}$ moves closer to the VBM where the - state dominates. These calculated hole concentrations are lower by about an order of magnitude than those seen in experiment [48,49]; the discrepancy may be due to unwanted impurities such as $\mathrm{C}$ that can be introduced during experimental growth, which are not accounted for here. $p_{0}$ and $\left[\mathrm{Ga}_{\mathrm{Sb}}\right]$ are also about an order of magnitude lower than those computed by Hakala et al. [56], which can be attributed to their lower value of $E_{f}\left(\mathrm{Ga}_{\mathrm{Sb}}^{2-}\right)$. The difference in formation energies is probably due to a combination of the difference in the functional and in the cruder image charge corrections used in their much earlier work. In Sb-rich conditions, we find that $E_{F}$ remains trapped at about $0.4 \mathrm{eV}$ above the VBM over the range of $T$ investigated due to the self-compensating defect physics, whereby the combined concentration of $\mathrm{Sb}_{\mathrm{Ga}}^{+}, \mathrm{Sb}_{\mathrm{Ga}}^{2+}$, and $\mathrm{Ga}_{i}^{+}$equals that of $V_{\mathrm{Ga}}^{-}, V_{\mathrm{Ga}}^{2-}$, and $\mathrm{Ga}_{\mathrm{Sb}}^{2-}$, with the individual proportions depending on $T$. Consequently, the electron concentration $n_{0}$ is equal to $p_{0}$, and the material is intrinsically insulating. This insulating nature is rarely seen experimentally; again, unwanted $p$-type impurities not included in this study, as well as nonequilibrium defect formation, expected to be important in samples grown epitaxially where kinetics dominate [16,49], may account for the discrepancy.

When imposing the charge neutrality constraint to determine the self-consistent $E_{F}$, it is possible to introduce fixed concentrations of other charged defects and calculate the equilibrium carrier and intrinsic defect concentrations in their presence. In this way, one can analyze compensation of fully ionized impurities in an approximate manner. By assuming a fixed concentration of some ionized donor, $\left[\mathrm{D}^{+}\right]=10^{18}$ $\mathrm{cm}^{-3}$, we have calculated donor compensation in $\mathrm{GaSb}$, with our results shown in Fig. 3(b). We find that, in Sb-poor conditions, rather than introducing $n$-type carriers, the donors are compensated by $\mathrm{Ga}_{\mathrm{Sb}}^{2-}$, so that $\left[\mathrm{D}^{+}\right]=2\left[\mathrm{Ga}_{\mathrm{Sb}}\right]$ for $T<$ $600 \mathrm{~K}$. We see, therefore, that in Sb-poor conditions donor doping will not be effective, assuming that defect formation occurs in equilibrium. In fact, $p_{0}$ will become greater than $10^{16} \mathrm{~cm}^{-3}$ at about $T=600 \mathrm{~K}$ and will continue to rise with temperature as $\left[\mathrm{Ga}_{\mathrm{Sb}}\right]$ increases above the value necessary to compensate $\left[\mathrm{D}^{+}\right]$due to thermal activation, while $E_{F}$ is pushed closer to the VBM. In Sb-rich conditions, however, we have $\left[\mathrm{D}^{+}\right]=n_{0}$ for most of the temperature range studied, so that $\mathrm{GaSb}$ will be doped effectively. At lower temperature, $E_{F}$ remains close to the CBM but decreases into the band gap with increasing temperature. There is a very small dip in $n_{0}$ around $T=400 \mathrm{~K}$, which occurs as thermally induced concentrations of $V_{\mathrm{Ga}}$ compensate the donors slightly. We note that, in MBE-grown samples intentionally doped to be $n$ type, increasing the $\mathrm{V} / \mathrm{III}$ ratio (i.e., going towards increasingly $\mathrm{Sb}$ rich conditions) caused a slight increase in compensating acceptor concentrations [51,52], contrary to our findings here. The effect is small and may be due to nonequilibrium defect formation and/or the presence of unwanted impurities.

In the same way, we can analyze acceptor compensation in GaSb. In Fig. 3(c), we show the equilibrium carrier and intrinsic defect concentrations in the presence of a fixed concentration of an ionized acceptor, $\left[\mathrm{A}^{-}\right]=10^{18} \mathrm{~cm}^{-3}$. The situation here is quite different from donor compensation discussed above; in both $\mathrm{Sb}$-poor and Sb-rich conditions the acceptors are uncompensated, and we have a $p$-type material with $p_{0}=\left[\mathrm{A}^{-}\right] . E_{F}$ remains close to the VBM but moves towards midgap as $T$ increases, as one would expect due to $T$-induced intrinsic carrier generation. In Sb-poor conditions, for $T>600 \mathrm{~K}$, substantial concentrations of $\mathrm{Ga}_{\mathrm{Sb}}$ form, which further contribute to the $p$-type activity. We therefore find that GaSb can be effectively $p$ doped, whether in Sbrich or Sb-poor conditions, a result that is consistent with experiment.

\section{Defects in InSb}

We show our calculated intrinsic defect formation energies as a function of $E_{F}$ referenced to the VBM in Fig. 4. We find that, in contrast to the case of $\mathrm{GaSb}$, we have a positively 


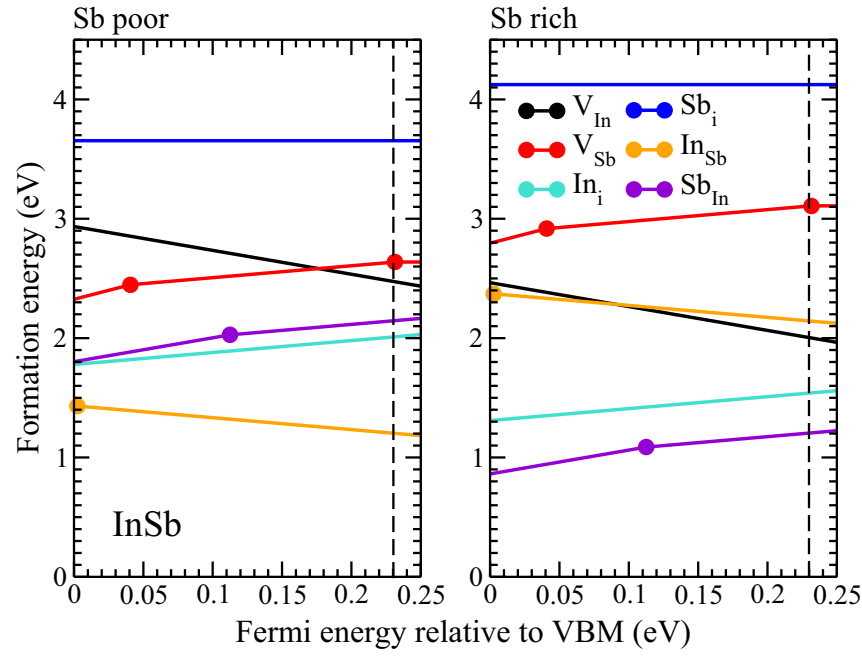

FIG. 4. Calculated formation energies of each intrinsic defect (vacancies, interstitials, and antisites; see text for description) in InSb as a function of Fermi energy relative to the valence band maximum (VBM), shown for Sb-poor and Sb-rich conditions. The slope of each line indicates the defect charge state; the transition levels lie where the slopes change. The dashed line indicates the position of the conduction band minimum.

charged defect, $\mathrm{Sb}_{\text {In }}$, dominating in Sb-rich conditions and a negatively charged defect, $\mathrm{In}_{\mathrm{Sb}}$, dominating in Sb-poor conditions. Consequently, one would expect an $n$-type material if grown in Sb-rich conditions and a (weakly, due to the relatively high formation energy) $p$-type material if grown in Sb-poor conditions. Experimentally, both $n$ - and $p$-type unintentionally doped samples are routinely prepared, and InSb can be doped relatively easily with electrons or holes as majority carriers [50,59-63]. Höglund et al. [64] calculated the defect formation energies using DFT-LDA, finding results consistent with ours for Sb-rich conditions, but for the Sb-poor conditions they found that $\mathrm{In}_{i}$ would dominate, resulting in an $n$-type material, in contrast to our results. In their calculations, they found InSb to be gapless, contradicting experiment, and did not discuss corrections for this error or for image charge interactions in their supercell model. The $\mathrm{Sb}_{\text {In }}$ defect has been proposed to be a source of intrinsic $n$-type carriers in epitaxially grown InSb but can be removed effectively by decreasing the $\mathrm{V} / \mathrm{III}$ ratio, i.e., moving away from Sb-rich conditions [63]. Such an observation is consistent with our calculated formation energies. Vacancies have also been proposed to be important in InSb [65,110-112], but our results show that their concentrations should be small as their formation energies are relatively high. We note that, although we have pointed out some differences between the defect physics of InSb and GaSb, some of these differences can be traced to the much lower band gap of InSb, compared with $\mathrm{GaSb}(0.23$ vs $0.808 \mathrm{eV})$. Restricting the range of $E_{F}$ to remain less than $0.23 \mathrm{eV}$ in GaSb would result in a transition level diagram similar to that of InSb. This result indicates a small valence band offset between the materials, consistent with earlier studies $[14,97,113]$.

As with the case of $\mathrm{GaSb}$, we have calculated equilibrium carrier and defect concentrations in InSb (excluding the

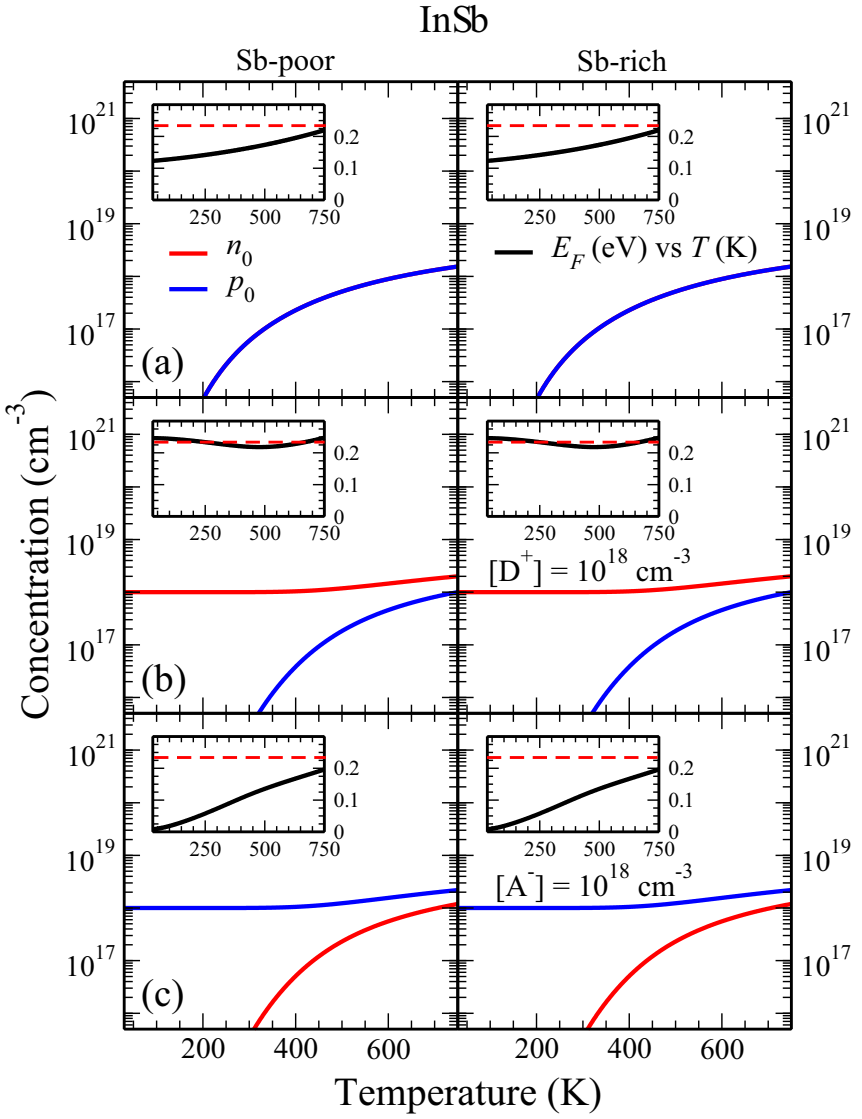

FIG. 5. Concentrations of electron $\left(n_{0}\right)$ and hole $\left(p_{0}\right)$ carriers and defects (vacancies, interstitials, and antisites; see text for description) in InSb as a function of temperature $T$ calculated (a) for equilibrium conditions, (b) in the presence of a fixed concentration of donors $\left[D^{+}\right]=10^{18} \mathrm{~cm}^{-3}$, and (c) for a fixed concentration of acceptors $\left[\mathrm{A}^{-}\right]=10^{18} \mathrm{~cm}^{-3}$. The results are shown for Sb-poor and Sb-rich conditions in the left and right panels, respectively. The insets show the self-consistent Fermi energy $E_{F}$ as a function of $T$, with the conduction band minimum indicated by the red dashed line.

variation in $E_{g}$ with $T$; see the discussion above); our results are shown in Fig. 5(a) over the $T$ range below the melting point (797 K [83]). Despite the dominance of positively and negatively charged defects in Sb-rich and Sb-poor conditions, respectively, we find that, under either condition InSb will be insulating as grown. This result is a consequence of the low band gap and relatively high defect formation energies; thermally induced intrinsic carrier formation will dominate as defect concentrations remain several orders of magnitude below the carrier concentrations over the relevant $T$ range (in Sb-poor conditions, $\left[\mathrm{In}_{\mathrm{Sb}}\right]$, not shown in the figure, rises above $10^{14} \mathrm{~cm}^{-3}$ only for $T>700 \mathrm{~K}$ ). $E_{F}$ remains closer to the CBM, as the DOS at the bottom of the conduction band is much lower than that at the top of the valence band. To produce $n$ - and $p$-type samples, therefore, one needs to dope the material, and nominally undoped samples that have substantial carrier concentrations probably have unwanted impurities present, according to our results.

In Fig. 5(b) we show the equilibrium carrier and defect concentrations in the presence of a fixed concentration of 
ionized donors, $\left[\mathrm{D}^{+}\right]=10^{18} \mathrm{~cm}^{-3}$. In both Sb-poor and $\mathrm{Sb}$-rich conditions, we find that $\mathrm{InSb}$ can be donor doped effectively, resulting in $n_{0}=\left[\mathrm{D}^{+}\right]$for much of the $T$ range. As the DOS is relatively low at the CBM, to induce the relevant electron concentration $E_{F}$ is pushed up to the CBM [see the inset in Fig. 5(b)]. No significant defect compensation is observed; indeed, we find that, for $T>400 \mathrm{~K}$, thermal ionization increases $n_{0}$ above $\left[\mathrm{D}^{+}\right]$.

We have also analyzed acceptor compensation in $\mathrm{InSb}$ by assuming a fixed ionized acceptor concentration, $\left[\mathrm{A}^{-}\right]=$ $10^{18} \mathrm{~cm}^{-3}$, and computing the resultant carrier and defect concentrations; our results are shown in Fig. 5(c). In both $\mathrm{Sb}$-poor and Sb-rich conditions there is no effective compensation of the acceptors by defects, indicating that InSb will be easily acceptor doped in either extreme condition. $E_{F}$ varies across the gap as $T$ increases, which induces minority-carrier concentrations while also increasing the majority-carrier concentration. We therefore see that InSb can be both $n$ and $p$ doped without significant compensation by intrinsic point defect formation, a result that is consistent with experiment $[50,63,64]$.

\section{SUMMARY}

We have investigated the intrinsic defect physics in $\mathrm{GaSb}$ and InSb by computing native defect formation energies using hybrid DFT. We justified our approach by first calculating a range of bulk properties of both systems, obtaining results in good agreement with experiment. We found that, in GaSb, $\mathrm{Ga}_{\mathrm{Sb}}$ will dominate in Sb-poor conditions, resulting in a $p$-type material, while in Sb-rich conditions selfcompensation will occur and the material will be intrinsic. We confirmed these inferences from the formation energy calculations by computing equilibrium carrier and defect concentrations as a function of temperature, then studying donor and acceptor compensation by assuming fixed concentrations of ionized dopants. We found that $\mathrm{GaSb}$ can be easily $p$ doped but, in equilibrium conditions, should only be effectively $n$ doped under Sb-rich conditions. For $\mathrm{InSb}$, we found that positively charged $\left(\mathrm{Sb}_{\text {In }}\right)$ and negatively charged antisite defects $\left(\mathrm{In}_{\mathrm{Sb}}\right)$ dominate in Sb-rich and $\mathrm{Sb}$-poor conditions, respectively. By calculating equilibrium carrier and defect concentrations, however, we showed that the material will be intrinsic as grown due to the relatively high formation energies, low band gap, and consequent thermally induced carrier generation. As the concentrations of compensating defects remain low over the relevant $T$ range, InSb can be effectively $n$ and $p$ doped. Our study provides crucial information on the defect physics of GaSb and InSb, important semiconductors for a range of technological applications.

\section{ACKNOWLEDGMENTS}

The authors acknowledge funding from EPSRC Grants No. ED/D504872, No. EP/K016288/1, and No. EP/I01330X/1 and the European Research Council (Grant No. 758345). The authors also acknowledge the use of the UCL Legion and Grace High Performance Computing Facilities (Legion@UCL and Grace@UCL) and associated support services, the IRIDIS cluster provided by the EPSRC funded Centre for Innovation (EP/K000144/1 and EP/K000136/1), the Thomas supercomputer via the U.K. Materials and Modelling Hub (EPSRC Grant No. EP/P020194/1), and the ARCHER supercomputer through membership of the UK's HPC Materials Chemistry Consortium, which is funded by EPSRC Grants No. EP/L000202 and No. EP/R029431, in the completion of this work.
[1] P. S. Dutta, H. L. Bhat, and V. Kumar, J. Appl. Phys. 81, 5821 (1997).

[2] A. Rogalski, J. Antoszewski, and L. Faraone, J. Appl. Phys. 105, 091101 (2009).

[3] P. Gogoi, D. Kamenskyi, D. D. Arslanov, R. T. Jongma, W. J. van der Zande, B. Redlich, A. F. G. van der Meer, H. Engelkamp, P. C. M. Christianen, and J. C. Maan, Phys. Rev. Lett. 119, 146603 (2017).

[4] S. Tomasulo, C. A. Affouda, N. A. Mahadik, M. E. Twigg, M. K. Yakes, and E. H. Aifer, J. Vac. Sci. Technol. B 36, 02D108 (2018).

[5] T. Ashley, A. B. Dean, C. T. Elliott, G. J. Pryce, A. D. Johnson, and H. Willis, Appl. Phys. Lett. 66, 481 (1995).

[6] C. A. Lehner, T. Tschirky, T. Ihn, W. Dietsche, J. Keller, S. Fält, and W. Wegscheider, Phys. Rev. Mater. 2, 054601 (2018).

[7] S. Petrosyan and A. Khachatryan, Semicond. Sci. Technol. 34, 045018 (2019).

[8] J. Paajaste, S. Suomalainen, R. Koskinen, A. Härkönen, M. Guina, and M. Pessa, J. Cryst. Growth 311, 1917 (2009).

[9] M. Guina, A. Rantamäki, and A. Härkönen, J. Phys. D 50, 383001 (2017).
[10] P. H. Jefferson, L. Buckle, B. R. Bennett, T. D. Veal, D. Walker, N. R. Wilson, L. F. J. Piper, P. A. Thomas, T. Ashley, and C. F. McConville, J. Cryst. Growth 304, 338 (2007).

[11] M. J. Ashwin, D. Walker, P. A. Thomas, T. S. Jones, and T. D. Veal, J. Appl. Phys. 113, 033502 (2013).

[12] H. Kala, G. A. Umana-Membreno, G. Jolley, N. D. Akhavan, M. A. Patrashin, K. Akahane, J. Antoszewski, and L. Faraone, Appl. Phys. Lett. 106, 032103 (2015).

[13] U. Serincan and B. Arpapay, Semicond. Sci. Technol. 34, 035013 (2019).

[14] L. Ley, R. A. Pollak, F. R. McFeely, S. P. Kowalczyk, and D. A. Shirley, Phys. Rev. B 9, 600 (1974).

[15] B. Shojaei, A. P. McFadden, M. Pendharkar, J. S. Lee, M. E. Flatté, and C. J. Palmstrøm, Phys. Rev. Mater. 2, 064603 (2018).

[16] M. Karalic, C. Mittag, M. Hug, T. Tschirky, W. Wegscheider, K. Ensslin, T. Ihn, K. Shibata, and R. Winkler, Phys. Rev. B 99, 115435 (2019). 
[17] G. A. Khodaparast, R. E. Doezema, S. J. Chung, K. J. Goldammer, and M. B. Santos, Phys. Rev. B 70, 155322 (2004).

[18] T. Campos, P. E. Faria Junior, M. Gmitra, G. M. Sipahi, and J. Fabian, Phys. Rev. B 97, 245402 (2018).

[19] K. L. Litvinenko, L. Nikzad, C. R. Pidgeon, J. Allam, L. F. Cohen, T. Ashley, M. Emeny, W. Zawadzki, and B. N. Murdin, Phys. Rev. B 77, 033204 (2008).

[20] V. Mourik, K. Zuo, S. M. Frolov, S. R. E. Plissard, P. A. M. Bakkers, and L. P. Kouwenhoven, Science 336, 1003 (2012).

[21] M. T. Deng, C. L. Yu, G. Y. Huang, M. Larsson, P. Caroff, and H. Q. Xu, Nano Lett. 12, 6414 (2012).

[22] T. Ashley, T. Burke, G. Pryce, A. Adams, A. Andreev, B. Murdin, E. O'Reilly, and C. Pidgeon, Solid-State Electronics 47, 387 (2003).

[23] T. D. Veal, L. F. J. Piper, S. Jollands, B. R. Bennett, P. H. Jefferson, P. A. Thomas, C. F. McConville, B. N. Murdin, L. Buckle, G. W. Smith, and T. Ashley, Appl. Phys. Lett. 87, 132101 (2005).

[24] P. H. Jefferson, T. D. Veal, L. F. J. Piper, B. R. Bennett, C. F. McConville, B. N. Murdin, L. Buckle, G. W. Smith, and T. Ashley, Appl. Phys. Lett. 89, 111921 (2006).

[25] L. Buckle, B. R. Bennett, S. Jollands, T. D. Veal, N. R. Wilson, B. N. Murdin, C. F. McConville, and T. Ashley, J. Cryst. Growth 278, 188 (2005).

[26] T. Ashley, L. Buckle, G. W. Smith, B. N. Murdin, P. H. Jefferson, L. F. J. Piper, T. D. Veal, and C. F. McConville, Proc. SPIE 6206, 62060L (2006).

[27] A. Belabbes, M. Ferhat, and A. Zaoui, Appl. Phys. Lett. 88, 152109 (2006).

[28] S. Iyer, L. Wu, J. Li, S. Potoczny, K. Matney, and P. R. C. Kent, J. Appl. Phys. 101, 113508 (2007).

[29] A. Lindsay, E. P. O'Reilly, A. D. Andreev, and T. Ashley, Phys. Rev. B 77, 165205 (2008).

[30] D. Wang, S. P. Svensson, L. Shterengas, G. Belenky, C. S. Kim, I. Vurgaftman, and J. R. Meyer, J. Appl. Phys. 105, 014904 (2009).

[31] M. J. Ashwin, T. D. Veal, J. J. Bomphrey, I. R. Dunn, D. Walker, P. A. Thomas, and T. S. Jones, AIP Adv. 1, 032159 (2011).

[32] V. Virkkala, V. Havu, F. Tuomisto, and M. J. Puska, Phys. Rev. B 85, 085134 (2012).

[33] J. J. Mudd, N. J. Kybert, W. M. Linhart, L. Buckle, T. Ashley, P. D. C. King, T. S. Jones, M. J. Ashwin, and T. D. Veal, Appl. Phys. Lett. 103, 042110 (2013).

[34] M. J. Ashwin, R. J. H. Morris, D. Walker, P. A. Thomas, M. G. Dowsett, T. S. Jones, and T. D. Veal, J. Phys. D 46, 264003 (2013).

[35] M. K. Rajpalke, W. M. Linhart, K. M. Yu, M. Birkett, J. Alaria, J. J. Bomphrey, S. Sallis, L. F. J. Piper, T. S. Jones, M. J. Ashwin, and T. D. Veal, Appl. Phys. Lett. 105, 212101 (2014).

[36] J. Buckeridge, D. O. Scanlon, T. D. Veal, M. J. Ashwin, A. Walsh, and C. R. A. Catlow, Phys. Rev. B 89, 014107 (2014).

[37] M. P. Polak, P. Scharoch, and R. Kudrawiec, Semicond. Sci. Technol. 30, 094001 (2015).

[38] W. M. Linhart, M. K. Rajpalke, J. Buckeridge, P. A. E. Murgatroyd, J. J. Bomphrey, J. Alaria, C. R. A. Catlow, D. O. Scanlon, M. J. Ashwin, and T. D. Veal, Appl. Phys. Lett. 109, 132104 (2016).
[39] E. P. O’Reilly, A. Lindsay, P. J. Klar, A. Polimeni, and M. Capizzi, Semicond. Sci. Technol. 24, 033001 (2009).

[40] T. F. Kuech, S. E. Babcock, and L. Mawst, Appl. Phys. Rev. 3, 040801 (2016).

[41] A. S. Chang, E. S. Zech, T. W. Kim, Y. H. Lin, L. J. Mawst, and R. S. Goldman, Appl. Phys. Lett. 105, 142105 (2014)

[42] M. Gladysiewicz, R. Kudrawiec, and M. S. Wartak, J. Appl. Phys. 119, 075701 (2016).

[43] Z. Song, S. Bose, W. Fan, D. H. Zhang, Y. Y. Zhang, and S. S Li, New J. Phys. 19, 073031 (2017).

[44] P. T. Webster, A. J. Shalindar, S. T. Schaefer, and S. R. Johnson, Appl. Phys. Lett. 111, 082104 (2017).

[45] K. Kharel and A. Freundlich, Semicond. Sci. Technol. 34, 055017 (2019).

[46] R. Jones-Albertus, E. Becker, R. Bergner, T. Bilir, D. Derkacs, O. Fidaner, D. Jory, T. Liu, E. Lucow, P. Misra et al., MRS Proc. 1538, 161 (2013).

[47] H. N. Leifer and W. C. Dunlap, Phys. Rev. 95, 51 (1954).

[48] Y. V. D. Meulen, J. Phys. Chem. Solids 28, 25 (1967).

[49] S. K. Haywood, A. B. Henriques, N. J. Mason, R. J. Nicholas, and P. J. Walker, Semicond. Sci. Technol. 3, 315 (1988).

[50] R. Pino, Y. Ko, and P. S. Dutta, High Performance Devices (World Scientific, Singapore, 2004), pp. 34-39.

[51] M. E. Lee, I. Poole, W. S. Truscott, I. R. Cleverley, K. E. Singer, and D. M. Rohlfing, J. Appl. Phys. 68, 131 (1990).

[52] G. W. Turner, S. J. Eglash, and A. J. Strauss, J. Vac. Sci. Technol. B 11, 864 (1993).

[53] C. C. Ling, M. K. Lui, S. K. Ma, X. D. Chen, S. Fung, and C. D. Beling, Appl. Phys. Lett. 85, 384 (2004).

[54] J. Kujala, N. Segercrantz, F. Tuomisto, and J. Slotte, J. Appl. Phys. 116, 143508 (2014).

[55] N. Segercrantz, I. Makkonen, J. Slotte, J. Kujala, T. D. Veal, M. J. Ashwin, and F. Tuomisto, J. Appl. Phys. 118, 085708 (2015).

[56] M. Hakala, M. J. Puska, and R. M. Nieminen, J. Appl. Phys. 91, 4988 (2002).

[57] A. Peles, A. Janotti, and C. G. Van de Walle, Phys. Rev. B 78, 035204 (2008).

[58] V. Virkkala, V. Havu, F. Tuomisto, and M. J. Puska, Phys. Rev. B 86, 144101 (2012).

[59] H. J. Hrostowski, F. J. Morin, T. H. Geballe, and G. H. Wheatley, Phys. Rev. 100, 1672 (1955).

[60] S. Zukotyński, S. Graf, and N. Saleh, Phys. Status Solidi B 42, K43 (1970).

[61] K. K. Chen and J. K. Furdyna, J. Appl. Phys. 43, 1825 (1972).

[62] M. Oszwalldowski and M. Zimpel, J. Phys. Chem. Solids 49, 1179 (1988).

[63] Y. Jin, D. Zhang, X. Chen, and X. Tang, J. Cryst. Growth 318, 356 (2011).

[64] A. Höglund, C. W. M. Castleton, M. Göthelid, B. Johansson, and S. Mirbt, Phys. Rev. B 74, 075332 (2006).

[65] X.-M. Zhao, Y. Zhang, L.-J. Cui, M. Guan, B.-Q. Wang, Z.-P. Zhu, and Y.-P. Zeng, Chin. Phys. Lett. 34, 076105 (2017).

[66] G. Kresse and J. Hafner, Phys. Rev. B 47, 558 (1993).

[67] G. Kresse and J. Hafner, Phys. Rev. B 49, 14251 (1994).

[68] G. Kresse and J. Furthmüller, Comput. Mater. Sci. 6, 15 (1996).

[69] G. Kresse and J. Furthmüller, Phys. Rev. B 54, 11169 (1996). 
[70] J. Heyd, G. E. Scuseria, and M. Ernzerhof, J. Chem. Phys. 124, 219906 (2006).

[71] P. E. Blöchl, Phys. Rev. B 50, 17953 (1994).

[72] D. Hobbs, G. Kresse, and J. Hafner, Phys. Rev. B 62, 11556 (2000).

[73] H. J. Monkhorst and J. D. Pack, Phys. Rev. B 13, 5188 (1976).

[74] M. Gajdoš, K. Hummer, G. Kresse, J. Furthmüller, and F. Bechstedt, Phys. Rev. B 73, 045112 (2006).

[75] J. Buckeridge, A. M. Teweldeberhan, and S. Fahy, Phys. Rev. B 79, 153201 (2009).

[76] J. Buckeridge and S. Fahy, Phys. Rev. B 84, 144120 (2011).

[77] J. Buckeridge, S. O'Halloran, and S. Fahy, Solid State Commun. 150, 1967 (2010).

[78] S. B. Zhang and J. E. Northrup, Phys. Rev. Lett. 67, 2339 (1991).

[79] C. Freysoldt, B. Grabowski, T. Hickel, J. Neugebauer, G. Kresse, A. Janotti, and C. G. Van de Walle, Rev. Mod. Phys. 86, 253 (2014).

[80] S. Lany and A. Zunger, Phys. Rev. B 78, 235104 (2008).

[81] T. R. Durrant, S. T. Murphy, M. B. Watkins, and A. L. Shluger, J. Chem. Phys. 149, 024103 (2018).

[82] J. Buckeridge, D. O. Scanlon, A. Walsh, and C. R. A. Catlow, Comput. Phys. Commun. 185, 330 (2014).

[83] D. R. Lide, editor, CRC Handbook of Chemistry and Physics, 89th ed. (CRC Press, Boca Raton, FL, 2008).

[84] If we were to use the experimental heats of formation, there would be no significant difference in our conclusions.

[85] J. Buckeridge, https://github.com/jbuckeridge/sc-fermi

[86] F. H. Taylor, J. Buckeridge, and C. R. A. Catlow, Chem. Mater. 28, 8210 (2016).

[87] J. Buckeridge, D. Jevdokimovs, C. R. A. Catlow, and A. A. Sokol, Phys. Rev. B 94, 180101(R) (2016).

[88] J. Buckeridge, Comput. Phys. Commun. (2019), doi: 10.1016/j.cpc.2019.06.017.

[89] N. N. Sirota and F. M. Gololobov, Dokl. Akad. Nauk SSSR 144, 398 (1962).

[90] H. J. McSkimin, A. Jayaraman, J. P. Andreatch, and T. B. Bateman, J. Appl. Phys. 39, 4127 (1968).
[91] W. F. Boyle and R. J. Sladek, Phys. Rev. B 11, 2933 (1975).

[92] M. Wu and C. Chen, J. Appl. Phys. 72, 4275 (1992).

[93] T. C. Chiang and D. E. Eastman, Phys. Rev. B 22, 2940 (1980).

[94] D. Hill and C. Schwerdtfeger, J. Phys. Chem. Solids 35, 1533 (1974).

[95] M. W. Heller and R. G. Hamerly, J. Appl. Phys. 57, 4626 (1985).

[96] K. Aoki, E. Anastassakis, and M. Cardona, Phys. Rev. B 30, 681 (1984).

[97] I. Vurgaftman, J. R. Meyer, and L. R. Ram-Mohan, J. Appl. Phys. 89, 5815 (2001).

[98] L. J. Slutsky and C. W. Garland, Phys. Rev. 113, 167 (1959).

[99] C. L. Littler and D. G. Seiler, Appl. Phys. Lett. 46, 986 (1985).

[100] W. Zawadzki, Adv. Phys. 23, 435 (1974).

[101] D. L. Price, J. M. Rowe, and R. M. Nicklow, Phys. Rev. B 3, 1268 (1971).

[102] G. P. Williams, F. Cerrina, G. J. Lapeyre, J. R. Anderson, R. J. Smith, and J. Hermanson, Phys. Rev. B 34, 5548 (1986).

[103] H. U. Middelmann, L. Sorba, V. Hinkel, and K. Horn, Phys. Rev. B 34, 957 (1986).

[104] J. W. Kim, S. Kim, J. M. Seo, S. Tanaka, and M. Kamada, J. Phys.: Condens. Matter 8, 4189 (1996).

[105] M. Shishkin and G. Kresse, Phys. Rev. B 74, 035101 (2006).

[106] M. Shishkin and G. Kresse, Phys. Rev. B 75, 235102 (2007).

[107] M. Shishkin, M. Marsman, and G. Kresse, Phys. Rev. Lett. 99, 246403 (2007).

[108] P. Y. Yu and M. Cardona, Fundamentals of Semiconductors, 3rd ed. (Springer-Verlag, Berlin, Heidelberg, 2005), Chap. 2.

[109] N. Segercrantz, J. Slotte, F. Tuomisto, K. Mizohata, and J. Räisänen, Phys. Rev. B 95, 184103 (2017).

[110] D. L. Kendall and R. A. Huggins, J. Appl. Phys. 40, 2750 (1969).

[111] A. N. Morozov, T. V. Abaeva, and V. T. Bublik, Cryst. Res. Technol. 21, 613 (1986).

[112] J. Xin, Q. Jiang, Y. Wen, S. Li, J. Zhang, A. Basit, L. Shu, X. Li, and J. Yang, J. Mater. Chem. A 6, 17049 (2018).

[113] R. Magri and A. Zunger, Phys. Rev. B 65, 165302 (2002). 